\title{
Aspectos generales en el diseño de políticas y programas de salud pública.
}

\author{
General features in public health policies and programs designing
}

Ernesto Cotonieto-Martínez ${ }^{a}$

\begin{abstract}
:
Mexico has increased its Gross Domestic Product (GDP) percent allocated for the health sector in recent years, however, this has been insufficient and doesn't comply the Pan American Health Organization (PAHO) recommendations. Therefore, the budget allocation has to give priority to policies and programs that optimize economic resources. The Logical Framework Approach-based (LFA) designs responds this need because it has elements that increase the probability of generating a positive impact on population health at low cost. The aim of this work is identify the most important features in the design process of public health policies and programs for the health professional to be able for planning interventions congruent with the economy, effectiveness and efficiency principles. Giving access for the design process participants to this type of resources will facilitate the making decisions and promote the use of scientific evidence-based information.
\end{abstract}

Keywords:

Public Policies, Public Health, Evidence-Based Practice.

\section{Resumen:}

México ha incrementado el porcentaje de su Producto Interno Bruto (PIB) destinado al sector salud en los últimos años, sin embargo, ha sido insuficiente y no cumple con lo recomendado por la Organización Panamericana de la Salud (OPS) ${ }^{1}$. Por lo tanto, la asignación del presupuesto debe priorizar a las políticas y programas que optimicen los recursos económicos. Los diseños basados en la Metodología del Marco Lógico (MML) atienden esta necesidad debido a que cuentan con elementos que incrementan la probabilidad de tener un impacto positivo en la salud de la población a bajo costo. El objetivo de este trabajo es identificar los aspectos más importantes en el diseño de políticas y programas públicos para que el profesional la salud sea capaz de plantear intervenciones congruentes con los principios de economía, eficacia y eficiencia. Dar acceso a este tipo de recursos a los participantes en el proceso de diseño, facilitará la toma de decisiones y promoverá el uso de información basada en evidencia científica.

\section{Palabras Clave:}

Políticas Públicas, Salud Pública, Práctica Basada en Evidencia.

\section{Introducción}

La MML es un proceso útil en el diseño, implementación y evaluación de intervenciones en el sector público (aunque también en la práctica privada). Durante la fase de diseño, su uso incrementa la posibilidad de que se contribuya a la creación del valor público a partir una política o programa presupuestario útil y potencialmente efectivo puesto que conlleva la sistematización de aspectos como:

\footnotetext{
- Análisis situacional

- Identificación del problema.

- Alineación estratégica a planes gubernamentales.

- Revisión de literatura y experiencias previas.
}

- Definición de una teoría del cambio.

- Delimitación de objetivos.

- Definición de supuestos (factores externos de riesgo)

- Elaboración de Matriz de Indicadores (con todo lo que implica).

Además, el uso de esta metodología permite legitimar las acciones gubernamentales, promover intervenciones basadas en evidencia científica e incrementar la calidad de vida de las personas, especialmente en materia de salud pública.

Finalmente, se debe señalar que el éxito del equipo de trabajo dependerá en gran medida de que se integren conocimientos $\mathrm{y} / \mathrm{o}$ experiencias respecto a tres áreas:

Autor de Correspondencia. Universidad Autónoma del Estado de Hidalgo. Instituto de Ciencias de la Salud. ORCID: 
proceso salud-enfermedad, métodos de investigación y evaluación de políticas y programas públicos.

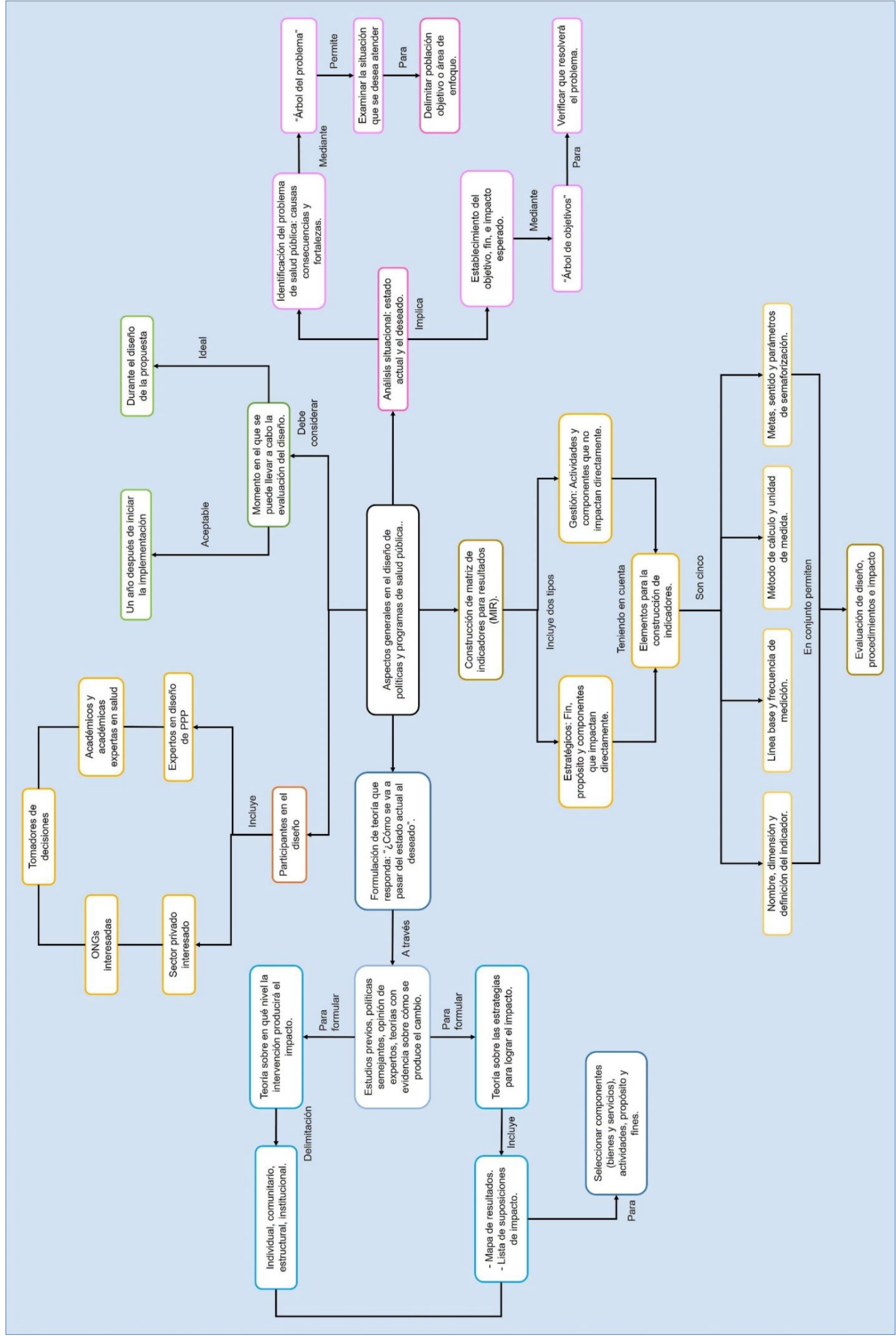




\section{Referencias}

[1] Organización Mundial de la Salud. Estrategias de Cooperación, OMS//OPS con México, Resumen. [Internet]. 2015. Disponible en: https://www.paho.org/mex/index.php?option=com_docman\&view=do wnload\&category_slug=technical-documentation\&alias $=1031-\mathrm{ccs}-$ brief-mex-esp2015\&Itemid=493

[2] Rogers P. La teoría del cambio, Síntesis metodológicas: evaluación de impacto $\mathrm{n}^{\circ} 2$ Centro de Investigaciones de UNICEF. [Internet]. 2014 Disponible en: https://www.unicefirc.org/publications/pdf/Brief\%202\%20Theory\%20of\%20Change_ES. pdf

[3] Maini R, Mounier-Jack S, \& Borghi J. How to and how not to develop a theory of change to evaluate a complex intervention: reflections on an experience in Democratic Republic of Congo. 2018. BMJ Glob Health 3(1), e000617. doi: 10.1136/bmjgh-2017-000617

[4] Organizational Research Services. Theory of Change: A practical Tool For Action, Results and Learning. [Internet]. 2004. Disponible en: https://www.aecf.org/resources/theory-of-change/

[5] Secretaría de Hacienda y Crédito Público. Guía para el diseño de indicadores estratégicos. [Internet]. 2016. Disponible en: https://www.gob.mx/cms/uploads/attachment/file/154446/Guia_Indica dores.pdf

[6] Sistema de Evaluación del Desempeño. Carga de Matriz de Marco Lógico. Manual del Usuario Versión 1.0. [Internet]. 2007. Disponible en: http://www.shcp.gob.mx/EGRESOS/PEF/sed/manual_sed.pdf 